typeset using JPSJ.sty $<$ ver.1.0b $>$

\title{
Correlation Effect on Peierls Transition
}

\author{
Muneo Sugiura 6 and Yoshikazu Suzumura ** \\ Department of Physics, Nagoya University, Nagoya 464-8602
}

(Received

)

\begin{abstract}
The effect of correlation on Peierls transition, which is accompanied by a dimerization, $t_{d}$, of a bond alternation for transfer energy, has been examined for a half-filled one-dimensional electron system with on-site repulsive interaction $(U)$. By applying the renormalization group method to the interaction of the bosonized Hamiltonian, the dimerization has been calculated variationally and self-consistently with a fixed electron-phonon coupling constant $(\lambda)$ and it is shown that $t_{d}$ takes a maximum as a function of $U$. The result is examined in terms of charge gap and spin gap and is compared with that of the numerical simulation by Hirsch [Phys. Rev. Lett 51 (1983) 296]. Relevance to the spin Peierls transition in organic conductors is discussed.
\end{abstract}

KEYWORDS: Peierls transition, Hubbard model, dimerization, renormalization, charge gap, spin gap

Peierls transition for a one-dimensional half-filled electron system coupled with phonon has been studied extensively since the Su-Schrieffer-Heeger model was proposed for the quasi-one-dimensional conductor polyacetylene with a bond alternation 1 ) The role of correlation in such a model has been examined by introducing an onsite repulsive interaction, $U$. The calculation using the Hartree-Fock approximation leads to the Peierls state, which exists only for $U$ smaller than a critical value of the order of the band width. ther developed by taking into account a one-dimensional quantum fluctuation. The numerical simulation exhibits an enhancement of dimerization in the presence of repulsive interaction 6 , 6. B. A notable finding is that the dimerization takes a maximum at a value of $U$ being nearly the band width becomes much larger than the dimerization gap.5) Regarding the case of weak coupling, the analytical method of a renormalization group (RG) has alsp exhibited the enhancement of $t_{d}$ as a function of $U$. 1 ) The effect of finite phonon frequency has been explored using the RG method based on the bosonization.11.12.13) It has been shown that the half-filled case leads to a competition between the state with both spin and charge gaps and the state with only a charge gap on the plane of the phonom frequency and the electron-phonon coupling constant.13) In contrast, the case of strong coupling with large $U$ has been examined in terms of the opposite approach, i.e., the expansion of $1 / U$ ) and mapping the electron system into a spin $1 / 2$ chain system, which leads to the spin Peierls transition. The successful treatment of the quantum fluctuation leads to the occurrence of the spin Peierls transition for an arbitrary magnitude of the electron-phonon coupling constant.14, 15, L6) However, it is not clear why the optimum condition for the Peierls transition is given by the intermediate coupling of $U$.

In the present paper, the unconventional role of $U$ on

*E-mail: sugiura@edu2.phys.nagoya-u.ac.jp

** E-mail: e43428a@nucc.cc.nagoya-u.ac.jp the Peierls state is examined by applying the RG method to the bosonized Hamiltonian. It is demonstrated that a maximum of the dimerization occurs for $U \simeq 2 t$ ( $4 t$ is the band width) for weak electron-phonon coupling $(\lambda)$, and that the charge gap is well separated from the spin gap at the maximum. Furthermore, we discuss the relevance of this to the spin Peierls transition in an organic conductor, $(\mathrm{TMTTF})_{2} \mathrm{PF}_{6}$ salt.17.18)

We consider a one-dimensional half-filled Hamiltonian given byㄴ. (2) B

$$
\begin{aligned}
H= & \sum_{j}\left[-\sum_{\sigma}\left(t-(-1)^{j} t_{d}\right)\left(c_{j, \sigma}^{\dagger} c_{j+1, \sigma}+h . c .\right)\right. \\
& \left.+U n_{j, \uparrow} n_{j, \downarrow}+C t_{d}^{2} / 2\right],
\end{aligned}
$$

where $c_{j, \sigma}^{\dagger}$ denotes a creation operator of a conduction electron with spin $\sigma(=\uparrow, \downarrow)$ at the lattice site $j$. The first term is the kinetic energy, where $t$ is the uniform transfer energy. The quantity, $t_{d}$, which denotes the dimerization due to Peierls distortion, is determined so as to minimize the total energy. The $U$ term with $n_{j, \sigma}=c_{j, \sigma}^{\dagger} c_{j, \sigma}$ denotes an on-site repulsive interaction, and the last term with a constant $C$ is the elastic energy for the distortion.

Applying the bosonization method 19.20) eq.(1) for the half-filled case is rewritten as

$$
\begin{aligned}
H= & +\frac{v_{\rho}}{4 \pi} \int d x\left[\frac{1}{K_{\rho}}\left(\partial_{x} \theta_{+}\right)^{2}+K_{\rho}\left(\partial_{x} \theta_{-}\right)^{2}\right] \\
& +\frac{v_{\sigma}}{4 \pi} \int d x\left[\frac{1}{K_{\sigma}}\left(\partial_{x} \phi_{+}\right)^{2}+K_{\sigma}\left(\partial_{x} \phi_{-}\right)^{2}\right] \\
& +\frac{v_{F}}{2 \pi \alpha^{2}} \int d x\left[y_{1 / 2} \cos 2 \theta_{+}+y_{\sigma} \cos 2 \phi_{+}\right. \\
& \left.-y_{W} \sin \theta_{+} \cos \phi_{+}+(1 / 8 \lambda) y_{W}^{2}\right],
\end{aligned}
$$

where $\theta_{ \pm}(x)$ and $\phi_{ \pm}(x)$ denote phase variaples for the charge and spin fluctuation, respectively 21 and $\left[\theta_{+}(x), \theta_{-}\left(x^{\prime}\right)\right]=\left[\phi_{+}(x), \phi_{-}\left(x^{\prime}\right)\right]=\mathrm{i} \pi \operatorname{sgn}\left(x-x^{\prime}\right)$. 
The $y_{1 / 2}$ term and the $y_{W}$ term represent the Umklapp scattering and the dimerization, respectively. The coefficients in eq.(2) are given by $K_{\rho}=(1+\tilde{U})^{-1 / 2}$, $K_{\sigma}=(1-\tilde{U})^{-1 / 2}, v_{\rho}=v_{\mathrm{F}}(1+\tilde{U})^{1 / 2}, v_{\sigma}=v_{\mathrm{F}}(1-\tilde{U})^{1 / 2}$, $y_{1 / 2}=y_{\sigma}=\tilde{U}, y_{W}=8 \alpha t_{d} / v_{\mathrm{F}}, 1 / \lambda=\pi v_{\mathrm{F}} C / 8 a, v_{\mathrm{F}}=$ $2 t a \sin k_{\mathrm{F}} a(=2 t a), k_{\mathrm{F}}=\pi /(2 a)$ and $\tilde{U}=U a /\left(\pi v_{\mathrm{F}}\right)$. The quantity $\alpha$ is a cutoff parameter of the order of the lattice constant, $a$; the ratio of $\alpha$ to $a$ will be evaluated later. The quantity $y_{W}$ is determined by minimizing the total energy, and the resultant self-consistency equation is written as

$$
\frac{1}{4 \lambda} y_{W}=\left\langle\sin \theta_{+} \cos \phi_{+}\right\rangle(\equiv \Delta),
$$

where $y_{W}=4 t_{d} \alpha /(t a)$. The quantity $\Delta$, which denotes an order parameter for the dimerization, is calculated self-consistently in terms of eq.(2). Equation (2) is examined using the RG method with a scaling $\alpha \rightarrow \alpha(1+d l)$. Within the lowest order of perturbation $\mathrm{RG}$ equations for the coupling constants are derived as $22,23,11,12,13,24$

$$
\begin{aligned}
\frac{d}{d l} K_{\rho}(l)= & -\frac{1}{2} y_{1 / 2}^{2}(l) K_{\rho}^{2}(l)-\frac{1}{16} y_{W}^{2}(l) K_{\rho}^{2}(l), \\
\frac{d}{d l} G_{\sigma}(l)= & -y_{\sigma}^{2}(l)-\frac{1}{8} y_{W}^{2}(l) \\
\frac{d}{d l} y_{1 / 2}(l)= & {\left[2-2 K_{\rho}(l)\right] y_{1 / 2}(l)+\frac{1}{8} y_{W}^{2}(l), } \\
\frac{d}{d l} y_{\sigma}(l)= & -G_{\sigma}(l) y_{\sigma}(l)-\frac{1}{8} y_{W}^{2}(l), \\
\frac{d}{d l} y_{W}(l)= & {\left[\frac{3}{2}-\frac{1}{2} K_{\rho}(l)-\frac{1}{4} G_{\sigma}(l)\right] y_{W}(l) } \\
& +\frac{1}{2} y_{1 / 2}(l) y_{W}(l)-\frac{1}{2} y_{\sigma}(l) y_{W}(l),
\end{aligned}
$$

where $G_{\sigma}(l)=2\left(K_{\sigma}(l)-1\right)$ and initial values at $l=0$ are given by coefficients of eq.(2). The relevance of $y_{1 / 2}$, $y_{\sigma}$ and $y_{W}$ corresponds to the appearance of charge gap, spin gap and dimerization, respectively.

For calculating the r.h.s. of eq.(3), we use the fact that the derivative of $\left\langle\sin \theta_{+} \cos \phi_{+}\right\rangle$with respect to $y_{W}$ can be expressed in terms of the response function given by

$$
R\left(\left|\vec{r}_{1}-\vec{r}_{2}\right|\right)=\left\langle T_{\tau} O_{d}\left(\vec{r}_{1}\right) O_{d}\left(\vec{r}_{2}\right)\right\rangle,
$$

where $O_{d}(\vec{r})\left(\equiv \sin \theta_{+}(\vec{r}) \cos \phi_{+}(\vec{r})=-(\pi \alpha / 4 a) \sum_{\sigma}(-1)^{j} c_{j}^{\dagger}\right.$ h.c.) denotes an operator for dimerization. The vector $\vec{r}$ consists of space $x(=j a)$ and imaginary time $\tau$ while $T_{\tau}$ is the time-ordering operator. Using the RG method, $R\left(t_{d}, l\right)(=R(|\vec{r}|))$ is calculated as 25$)$

$$
\begin{aligned}
R\left(t_{d}, l\right)= & \frac{1}{4} \exp \left[-\int_{0}^{l} \mathrm{~d} l^{\prime}\left(K_{\rho}\left(l^{\prime}\right)\right.\right. \\
& \left.\left.+K_{\sigma}\left(l^{\prime}\right)-y_{1 / 2}\left(l^{\prime}\right)+y_{\sigma}\left(l^{\prime}\right)\right)\right], \quad\left(l \leq l_{c}\right) \\
R\left(t_{d}, l\right)= & \frac{1}{4} \exp \left[-\int_{0}^{l_{c}} \mathrm{~d} l^{\prime}\left(K_{\rho}\left(l^{\prime}\right)\right.\right. \\
& \left.\left.+K_{\sigma}\left(l^{\prime}\right)-y_{1 / 2}\left(l^{\prime}\right)+y_{\sigma}\left(l^{\prime}\right)\right)\right] \\
& \times \exp \left[-\int_{l_{c}}^{l} \mathrm{~d} l^{\prime}\left(K_{\sigma}\left(l^{\prime}\right)+y_{\sigma}\left(l^{\prime}\right)\right)\right], \quad\left(l>l_{c}\right)
\end{aligned}
$$

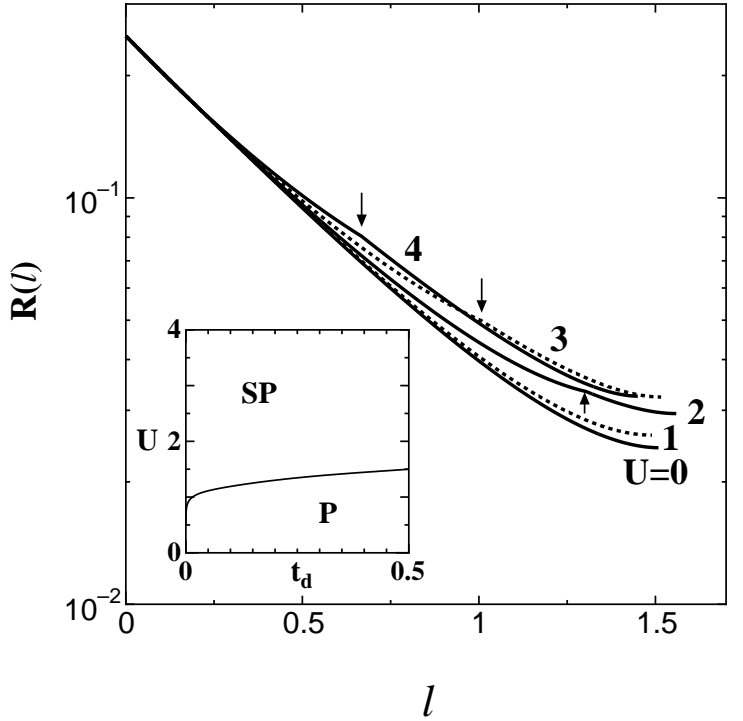

Fig. 1. $l$-dependence of $R(l)$ of eq.(6) for $t_{d}=0.1$, and $U=0$, $1,2,3$ and 4 where $R(l)$ decreases and takes a minimum at $l=l_{m} . \quad t=1$ and $\alpha / a=1.52$. The arrow denotes $l=l_{c}$ given by $y_{1 / 2}\left(l_{c}\right)=1$. In the inset, the spin Peierls (SP) region and the Peierls $(\mathrm{P})$ region correspond to $l_{c}<l_{m}$ and $l_{c}>l_{m}$, respectively, where the boundary denotes a crossover between these two regions.

where $l=\ln \left(\left[x^{2}+\left(v_{F} \tau\right)^{2}\right]^{1 / 2} / \alpha\right)$. In eq.(6), we introduced $l=l_{c}$ with $y_{1 / 2}\left(l_{c}\right)=1$, from which the charge gap is calculated. The response function for $l>l_{c}$ is calculated by estimating $\left\langle\sin \theta_{+}\right\rangle$in the presence of the charge gap. Although one expects $R(\infty) \rightarrow \Delta^{2}$, we use the following method to estimate $\Delta$ within the present RG equation, which leads to $R(l)$ taking a minimum at $l=l_{m}$. Since $R(l)$ as a function of $l$ becomes invalid for $l>l_{m}$, we use a self-consistency equation for $\Delta\left(=\Delta\left(t_{d}\right)\right)$ given by

$\Delta\left(t_{d}\right)=\int_{0}^{t_{d}} \mathrm{~d} t_{d}^{\prime} \int_{0}^{l_{m}\left(t_{d}^{\prime}\right)} \mathrm{d} l^{\prime} e^{2 l^{\prime}}\left\{R\left(t_{d}^{\prime}, l^{\prime}\right)-\Delta^{2}\left(t_{d}^{\prime}\right)\right\}(7$.

We assume $t=1$ in the following numerical calculation, if there is no confusion. For estimating the r.h.s. of eq.(7), we calculate RG eq.(4). When $l$ increases with of $(\bar{x}) \mathrm{d}_{j} U_{1}(\curvearrowright)(0)$, one finds the decrease of $K_{\rho}(l), G_{\sigma}(l)$ and $y_{\sigma}(l)$ and the increase of $y_{1 / 2}$ and $y_{W}$. Behaviors at large $l$ are classified into two groups depending on the magnitude of $y_{W}(0)$. For small $y_{W}(0), y_{1 / 2}(l)$ is larger than $y_{W}(l)$, while $y_{1 / 2}(l)$ becomes smaller than $y_{W}(l)$ with increasing $y_{W}(0)$. The former case denotes the Peierls state in the presence of a well-developed charge gap since the relevance of $y_{1 / 2}\left(y_{W}\right)$ corresponds to the formation of the charge gap (the formation of both charge and spin gaps due to the Peierls distortion).

The response function is calculated by substituting the solution of RG equations into eq.(6). In Fig.1, the $l$ dependence of $R(l)$ is shown for $t_{d}=0.1$ and certain values of $U$. The results are shown in the region with $l<l_{m}$, where $R\left(l_{m}\right)$ is a minimum of $R(l)$. The long range order of the Peierls state is related to the relevance of $y_{W}$, which leads to the appearance of $l_{m}$ in the present approximation of the RG equations. The arrow 
for $U=2,3$ and 4 denotes the location at $l=l_{c}$ where $y_{1 / 2}\left(l_{c}\right)=1$. From a comparison of $l_{c}$ with $l_{m}$, one finds a distinction between case I $\left(l_{m}>l_{c}\right)$ and case II ( $\left.l_{m}<l_{c}\right)$. The charge gap is already developed prior to obtaining the Peierls state in case I. The inset depicts the spin Peierls region (case I) and the Peierls region (case II) on the plane of $U$ and $t_{d}$. The charge gap dominates for $U \gtrsim 1.5$ and $t_{d}<0.5$. The solid curve shows the boundary as a crossover between these two regions, where the state moves continuously.

An estimation of the ratio of $\alpha / a$ is needed for the calculation of $\Delta$ of eq.(7) as a function of $t_{d}$ and $U$. When $U=0, \Delta$ becomes equal to the order parameter of the conventional Peierls distortion and thus the r.h.s. of eq.(3) can be calculated rigorously as $\Delta \rightarrow \Delta_{\text {Peierls }}=\left(t_{d} \alpha / a\right) \int_{0}^{\pi} \mathrm{d} z(\sin z)^{2} /\left[(2 \cos z)^{2}+\right.$ $\left.\left(2 t_{d} \sin z\right)^{2}\right]^{1 / 2}$, which leads to $\left(t_{d} \alpha / a\right) \times \ln \left(1.4 / t_{d}\right)$ for small $t_{d}$. By comparing $\Delta_{\text {Peierls }}$ with that obtained from eq.(7), we obtain $\alpha / a \simeq 1.5 \pm 0.02$ for small $t_{d}(\lesssim 0.2)$.

In terms of the response function, $\Delta$ is calculated self-

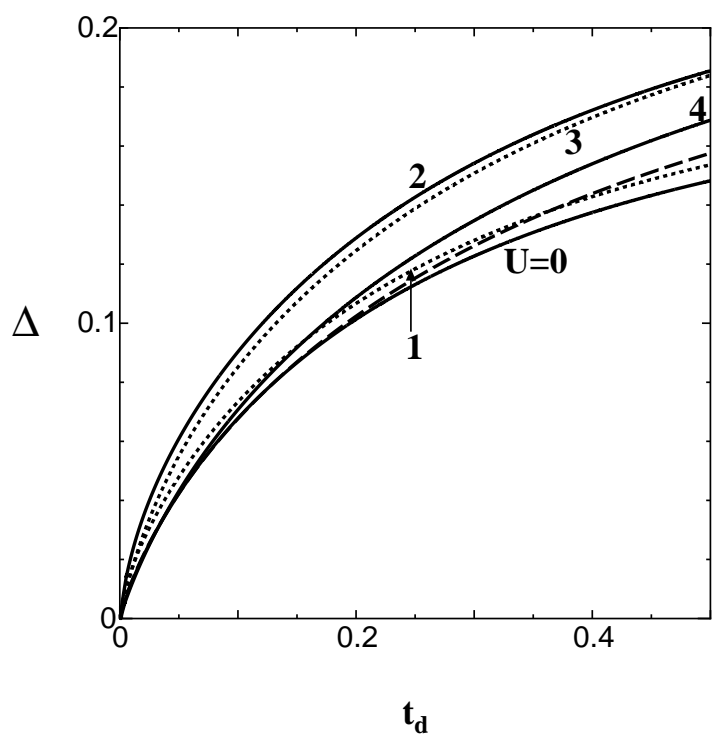

Fig. 2. $t_{d}$-dependence of $\Delta$ calculated self-consistently by using eq.(7) for $U=0,1,2,3$ and 4 . The dashed curve denotes $\Delta_{\text {Peierls }}$.

consistently from eq. (7). In Fig. $2, \Delta$ is shown as a function of $t_{d}$ for certain values of $U$. The dashed curve denotes $\Delta_{\text {Peierls }}$ which coincides well with that of $U=0$ (solid curve) for $t_{d} \lesssim 0.2$. With increasing $t_{d}, \Delta$ for fixed $U$ increases monotonically. The self-consistent result of $\Delta$ obtained from eq. (7) is smaller than $R\left(l_{m}\right)^{1 / 2}$, e.g., $\Delta / R\left(l_{m}\right)^{1 / 2}=0.43,0.45,0.52,0.47$ and 0.39 for $U=0$ ,1, 2, 3 and 4 . This is reasonable since the lowest order $\mathrm{RG}$ equation with the relevant coupling overestimates the response function for large $l$. From the comparison of $\Delta$ for $U=2$ with that for $U=3$, it is found that $\Delta$ as a function of $U(\gtrsim 2)$ decreases, indicating the existence of an optimum value of $U$ for the Peierls state.

Based on Fig. 2, we obtain the dimerization, $t_{d}$, representing the Peierls state, which is calculated using eq.(3). In Fig. $3, t_{d}$ is shown as a function of $\lambda$ with certain values of $U$. The dashed curve denotes $t_{d}(\simeq 1.4 \exp [-1 / \lambda])$

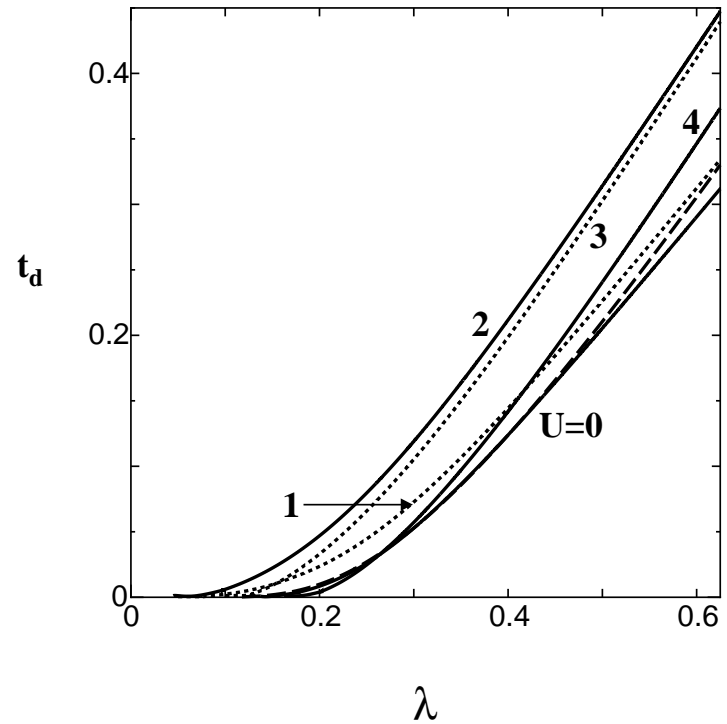

Fig. 3. $\lambda$-dependence of $t_{d}$, which is calculated from eq.(3), for $U=0,1,2,3$ and 4 where the dashed curve denotes $\Delta_{\text {Peierls }}$ corresponding to the conventional calculation in the absence of $U$.

obtained from $\Delta_{\text {Peierls, }}$, which coincides with $t_{d}$ in the case of $U=0$ (solid curve) for $0.25 \lesssim \lambda \lesssim 0.5$ within the visible scale. For small $\lambda, t_{d}$ decreases exponentially but is enhanced sufficiently for $U=1,2$ and 3 . We note that $t_{d}$ takes a maximum around $U \simeq 2$. Such a $U$ dependence of $t_{d}$, indicating a crossover from the regime of weak coupling to that of strong coupling, is elucidated by examining the relevant term of $y_{W} \sin \theta_{+} \cos \phi_{+}$in eq.(2) and the corresponding RG equation (the last equation of eq.(4)). For small $U$, the $y_{W}$-term is enhanced by the Umklapp scattering of the $y_{1 / 2}$-term, which leads to the suppression of charge fluctuation $\left(\theta_{+}\right)$. For large $U$, both $y_{\sigma}$ and $K_{\sigma}$ increase the quantum fluctuation for the spin part $\left(\phi_{+}\right)$resulting in the suppression of $\left\langle\cos \phi_{+}\right\rangle$ for the Peierls state.

The $U$-dependence of $t_{d}(=\Delta \lambda a / \alpha)$ is obtained by examining $t_{d}$ with fixed $\lambda$ in Fig. 3. In Fig. 4, $t_{d}$ with $\lambda=0.25$ is depicted as a function of $U$ by the solid curve. As expected from Fig. 2 and Fig. $3, t_{d}$ takes a maximum around $U=2$. The triangle, which represents the numerical result obtained by Hirsch, 5 is compared with the dashed curve of the present calculation with $\lambda=0.42$ (dashed curve) corresponding to the parameter of the numerical simulation. The fact that the dashed curve is larger than the numerical result (triangle) is reasonable since the present calculation treats the dimerization ( the bond alternation) classically while the numerical simulation has been performed in the presence of the quantum fluctuation. We note that there is not much variation of the location of $U$ for the maximum, e.g., the maximum appears at $U=2.2 \pm 0.1$ in the interval range of $0.2 \lesssim \lambda \lesssim 0.5$. In the inset, the $U$-dependences of the charge gap $\left(\Delta_{\rho}\right)$ and spin gap $\left(\Delta_{\sigma}\right)$ for $\lambda=0.25$ are shown by a solid curve and dotted curve, respectively. These gaps are calculated by $\Delta_{\rho} / \omega_{c}=\exp \left[-l_{c}\right]$ and $\Delta_{\sigma} / \omega_{c}=\exp \left[-l_{\sigma}\right]$ with $\left|y_{\sigma}\left(l_{\sigma}\right)\right|=1$, where $\omega_{c}(\simeq 2.37)$ 


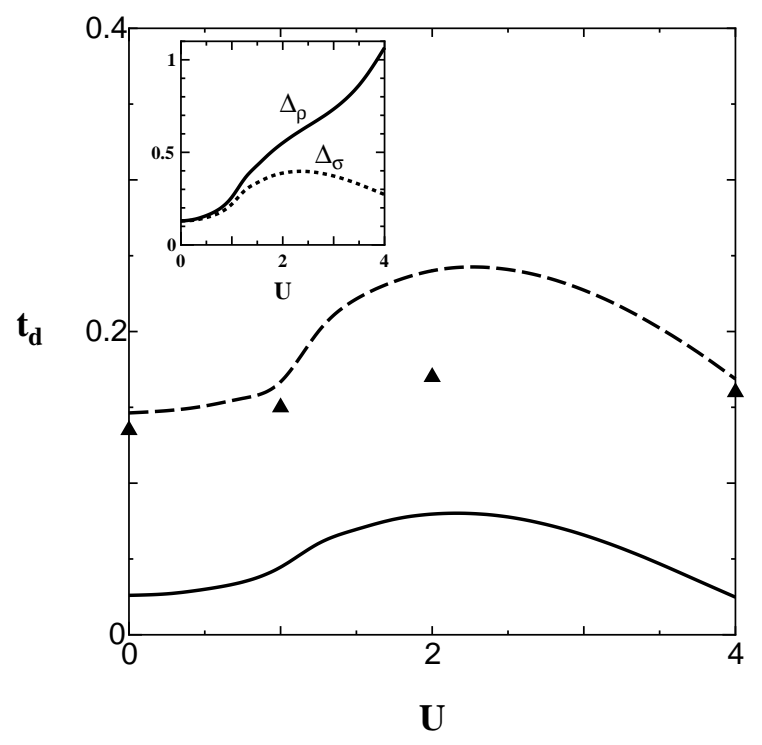

Fig. 4. Quantity $t_{d}$ as a function of $U$ for $\lambda=0.25$ (solif curve). The symbol (triangle) is the result calculated by Hirsch 5 which is compared with the present calculation with $\lambda=0.42$. The inset shows the charge gap $\left(\Delta_{\rho}\right)$ and the spin gap $\left(\Delta_{\sigma}\right)$ for $\lambda=$ 0.25 .

is chosen so as to obtain $\Delta_{\rho}$ with $t_{d}=0$ nearly equal to the well-known exact one. With increasing $U, \Delta_{\rho}$ increases monotonically while $\Delta_{\sigma}$ takes a maximum. The maximum of $t_{d}$ is found to be accompanied by the separation of charge gap and spin gap. Thus, it is expected that with increasing $U$, there is a crossover from the conventional Peierls state with $\Delta_{\rho} \simeq \Delta_{\sigma}$ to the spin Peierls state with the charge gap being much larger than the spin gap.

When $U$ is extremely large, the effect of $t_{d}$ can be examined $\mu$ sing the bosonized phase Hamiltonian of spin $1 / 2$ chain 16 with the antiferromagnetic exchange energy, $4\left(t \pm t_{d}\right)^{2} / U$. The calculation of $t_{d}$, similar to eq.(3), with only a spin degree of freedom shows that $t_{d}$ decreases monotonically with increasing $U$. From comparison of the present result with this limiting one (not shown explicitly), the present calculation for $U \lesssim 4$ in Fig. 3 seems to be reasonably extrapolated to that of the spin $1 / 2$ chain except for small $t_{d}$ (and then small $\lambda$ ), which requires much accuracy of numerical evaluation.

Finally, we discuss the spin Peierls transition in an organic conductor, (TMTTF $)_{2} \mathrm{PF}_{6}$, which has peen observed by magnetic and X-ray experiments.26.27) This conductor, which indicates the charge gap being much larger than the spin gapts) is often analyzed in terms of a model with spin $1 / 2$ chain although $U$ is of the order of the band width.28 The conductor has a quarter-filled band with a dimerization, which may be considered to be half-filling. However, such an effectively half-filled band strongly reduces the magnitude of Umklapp scattering, resulting in the suppression of the Peierls state. In fact, the Umklapp scattering is estimated to be $y_{1 / 2}=$ $2\left(x_{d} /\left(1+x_{d}^{2}\right)\right) \tilde{U}$, where $v_{\mathrm{F}}=\sqrt{2} t a$ and $x_{d}$ corresponds to a dimerization for a quarter-filling.29) For $x_{d} \simeq 0.1$ corresponding to TMTTF salt, 17) we find that $t_{d}$ as a function of $U$ decreases for small $U$ while separation between $\Delta_{\rho}$ and $\Delta_{\sigma}$ appears for $U \gtrsim 3$. Moreover, with decreasing $x_{d}$ and fixed $\lambda(\lesssim 0.5), t_{d}$ decreases strongly for $U=4 \sim 6$. When $U=5.6$ and $\lambda=0.25(0.5)$, we obtain the dimerization as $t_{d}=0.036(0.23), 0.019(0.17)$ and $\simeq 0(0.05)$; the charge gap as $\Delta_{\rho}=0.31(1.05), 0.12(0.73)$ and $\simeq$ 0 (0.17); the spin gap as $\Delta_{\sigma}=0.18(0.77), 0.08(0.57)$ and $\simeq 0(0.15)$, for $x_{d}=0.2,0.1$ and 0 , respectively. Based on such a consideration, the spin Peierls state in the organic conductor could be realized when Umklapp scattering induced by the electronic correlation becomes large.

In summary, we have examined the effect of on-site repulsive interaction, $U$, on the Peierls state with dimerization $t_{d}$. For small (large) $U, t_{d}$ increases (decreases) where the charge gap is almost equal to (much larger than) the spin gap. The maximum of $t_{d}$ indicates a crossover from the weak coupling regime, in which the Umklapp scattering suppresses the charge fluctuation, into a strong coupling regime, in which the quantum spin fluctuation reduces the effect of dimerization.

[1] W.P. Su, J.R. Schrieffer and A.J. Heeger: Phys. Rev. Lett 42 (1979) 1698.

[2] K.R. Subbaswamy and M. Grabowski: Phys. Rev. B 24 (1981) 2168

[3] S. Kivelson and D.E. Heim: Phys. Rev. B 26 (1982) 4278.

[4] S. Mazumdar and S.N. Dixit: Phys. Rev. Lett 51 (1983) 292.

[5] J.E. Hirsch: Phys. Rev. Lett 51 (1983) 296.

[6] S.N. Dixit and S. Mazumdar: Phys. Rev. B 29 (1984) 1824.

[7] Z.G. Soos and S. Ramasesha: Phys. Rev. B 29 (1984) 5410.

[8] D. Baeriswyl and K. Maki: Phys. Rev. B 31 (1985) 6633.

[9] G.W. Hyden and E.J. Mele: Phys. Rev. B 32 (1985) 6527.

[10] B. Horovitz and J. Sólyom: Phys. Rev. B 32 (1985) 2681.

[11] J. Voit and H.J. Schulz: Phys. Rev. B 37 (1988) 10068.

[12] J. Voit: Phys. Rev. Lett 64 (1990) 323.

[13] K. Yonemitsu and M. Imada: Phys. Rev. B 54 (1996) 2410.

[14] M.C. Cross and D.S. Fisher: Phys. Rev. B 19 (1979) 402.

[15] T. Nakano and H. Fukuyama: J. Phys. Soc. Jpn. 49 (1980) 1679.

[16] S. Inagaki and H. Fukuyama: Kotaibutsuri 20 (1985) 369 [in Japanese].

[17] T. Ishiguro and K. Yamaji: Organic Superconductors (Springer-Verlag, Berlin, 1990).

[18] K. Bechgaard and D. Jérome: Physica. Scripta. T39 (1991) 37.

[19] A. Luther and I. Peschel: Phys. Rev. B 9 (1974) 2911.

[20] D.C. Mattis and E.H. Lieb: J. Math. Phys. 6 (1965) 304.

[21] Y. Suzumura: Prog. Theor. Phys. 61 (1979) 1.

[22] J. Sólyom: Adv. Phys. 28 (1979) 201.

[23] T. Giamarchi and H.J. Schulz: J. Phys. France 49 (1988) 819.

[24] M. Tsuchiizu and Y. Suzumura: J. Phys. Soc. Jpn. 68 (1999) 3966.

[25] T. Giamarchi and H.J. Schulz: Phys. Rev. B 39 (1989) 4620.

[26] J.P. Pouget, R. Moret, R. Comes, K. Bechgaard, J.M. Fabre and L. Giral: Mol. Cryst. Liq. Cryst. 79 (1982) 129.

[27] M. Dumm, M. Dressel, A. Loidl, B.W. Frawel, K.P. Starkey and L.K. Montgomery: Synth. Met 103 (1999) 2068.

[28] F. Mila: Phys. Rev. B 52 (1995) 4788.

[29] M. Tsuchiizu, H. Yoshioka and Y. Suzumura: J. Phys. Soc. Jpn. 68 (1999) 1809 